\title{
CONFORMAL MAPPING OF REGIONS BOUNDED BY CURVILINEAR POLYGONS
}

\author{
BURTON RODIN ${ }^{1}$
}

\begin{abstract}
The main result is the proof that, under a natural notion of conformal equivalence, any curvilinear polygon is conformally equivalent to a curvilinear polygon whose inside region is starlike. The method of proof is a new type of continuity argument which, in distinction to the classical continuity method, yields existence without requiring uniqueness.
\end{abstract}

The theorem proved in this paper can be described roughly as follows: Any curvilinear polygonal region in the plane can be mapped conformally onto a starlike curvilinear polygonal region in such a manner that the continuous extension of the mapping to the boundaries sends vertices to vertices and preserves vertex angles.

This problem was brought to my attention by Professor William Helton. The method of solution involves a continuity argument substantially different from the classical one which requires uniqueness of the conformal mapping, at least locally in parameter space [3, p. $234 \mathrm{ff}$.].

A piecewise $C^{1}$ closed Jordan curve in the plane will be called a curvilinear polygon. A curvilinear polygon determines a cyclic sequence of class $C^{1}$ arcs which are disjoint except for their endpoints. We call the $C^{1}$ arcs edges, and the endpoints vertices. Each vertex angle may be measured by a real number $\alpha,-\pi \leq \alpha \leq \pi$, which describes the change in direction of the positively oriented tangent vector at the vertex; $\alpha=0$ describes a vertex at which $\gamma$ possesses a tangent, $\alpha=\pi$ describes a protruding cusp with interior angle $0^{\circ}, \alpha=-\pi$ describes an intruding cusp with interior angle $360^{\circ}$.

Let $\rho$ and $\sigma$ be curvilinear polygons. We say $\rho$ and $\sigma$ are conformally equivalent if there is a 1-1 conformal map $F: R \rightarrow S$ of the inside of $\rho$ onto the inside of $\sigma$ such that: (i) $F$ can be extended to a homeomorphism $\tilde{F}: \mathrm{Cl} R \rightarrow \mathrm{Cl} S$ which makes the vertices of $\rho$ and $\sigma$ correspond, and (ii) the vertex angles at corresponding vertices are equal.

An equivalent condition can be found using the classical Lindelöf theorem $[5, \mathrm{p}$. 358]: $\rho$ and $\sigma$ are conformally equivalent if and only if there is a 1-1 conformal map $F$ of the inside of $\rho$ onto the inside of $\sigma$ such that $\arg F^{\prime}$ has a continuous extension to $\rho$.

THEOREM. Any curvilinear polygon is conformally equivalent to a curvilinear polygon whose inside region is starlike.

REMARK. This theorem remains true if the definition of curvilinear polygon is modified by requiring that the edges be of class $C^{k}(k=2,3, \ldots, \infty)$ instead of

Received by the editors May 18, 1984.

1980 Mathematics Subject Classification. Primary 30A30.

${ }^{1}$ Research supported in part by the National Science Foundation.

(c) 1985 American Mathematical Society $0002-9939 / 85 \$ 1.00+\$ .25$ per page 
$C^{1}$. The proof would have to be modified only in that the small circles used in the construction of $\gamma$ (Figure 1) would have to be replaced by closed curves having higher order contract with the unit circle.

ProOF. Let $\rho$ be a given curvilinear polygon. Let $R$ be its inside region and let $z_{0} \in R$. We shall construct a region $S$, starlike with respect to the origin, such that $\sigma=\partial S$ is a curvilinear polygon conformally equivalent to $\rho$. Moreover, there is a 1-1 conformal mapping of $R$ onto $S$ which exhibits the conformal equivalence and which sends the prescribed point $z_{0}$ to the origin.

Let $r_{0}, r_{1}, \ldots, r_{n}$ be the vertices of $\rho$ in positive cyclic order and let $\alpha_{0}, \alpha_{1}, \ldots, \alpha_{n}$ be the corresponding vertex angles. Let $g$ be the Riemann mapping function of $R$ onto the unit disk, normalized so that $g\left(z_{0}\right)=0$ and $\tilde{g}\left(r_{0}\right)=1$, where $\tilde{g}$ is the continuous extension of $g$ to $\rho$. For $k=1,2, \ldots, n$ determine $\theta_{k} \in(0,2 \pi)$ by $\tilde{g}\left(r_{k}\right)=e^{i \theta_{k}}$. Note that $0<\theta_{1}<\theta_{2}<\cdots<\theta_{n}<2 \pi$.

We shall construct a curvilinear polygon $\gamma$, starlike with respect to the origin, and with vertex angles $\alpha_{0}, \alpha_{1}, \ldots, \alpha_{n}$. The construction of $\gamma$ will depend on $n$ real parameters. In fact, each point $\vec{\varphi}=\left(\varphi_{1}, \varphi_{2}, \ldots, \varphi_{n}\right)$ in the open $n$-simplex

$$
E_{n}=\left\{\left(\varphi_{1}, \varphi_{2}, \ldots, \varphi_{n}\right) \in \mathbf{R}^{n}: 0<\varphi_{1}<\varphi_{2}<\cdots<\varphi_{n}<2 \pi\right\}
$$

will determine such a unique $\gamma$ which we denote by $\gamma=\gamma(\vec{\varphi})$. Suppose for the time being that we have defined $\gamma(\vec{\varphi})$ for each $\vec{\varphi} \in E_{n}$ (the exact construction, which is rather arbitrary, will be described later).

Let $\vec{\varphi} \in E_{n}$. Denote the vertices of $\gamma(\vec{\varphi})$ by $\varsigma_{0}, \varsigma_{1}, \ldots, \varsigma_{n}$. Consider the Riemann mapping function of the inside of $\gamma(\vec{\varphi})$ onto the unit disk, normalized so that it sends the origin to the origin and so that its continuous extension to $\gamma(\vec{\varphi})$ sends $\zeta_{0}$ to 1 . This extension sends $\zeta_{1}, \zeta_{2}, \ldots, \zeta_{n}$ to points $e^{i t_{1}}, e^{i t_{2}}, \ldots, e^{i t_{n}}$ with $0<$ $t_{1}<t_{2}<\cdots<t_{n}<2 \pi$. In this way we associate $\left(t_{1}, t_{2}, \ldots, t_{n}\right) \in E_{n}$ with $\vec{\varphi}=\left(\varphi_{1}, \varphi_{2}, \ldots, \varphi_{n}\right) \in E_{n}$. This association, denoted $\vec{t}=T(\vec{\varphi})$, gives a map $T: E_{n} \rightarrow E_{n}$. If we show that $T$ is surjective, the theorem will be proved. Indeed, if $\vec{\theta}=\left(\theta_{1}, \theta_{2}, \ldots, \theta_{n}\right)$ is the vector in $E_{n}$ defined in the first paragraph of the proof and if $T(\vec{\psi})=\vec{\theta}$ for some $\vec{\psi} \in E_{n}$, then $\gamma(\vec{\psi})$ can be taken as the $\sigma$ in the statement of the theorem.

We shall show that $T: E_{n} \rightarrow E_{n}$ is surjective by proving the following two assertions:

Assertion 1. $T: E_{n} \rightarrow E_{n}$ is continuous.

Assertion 2. $T$ has a continuous extension $\tilde{T}: \bar{E}_{n} \rightarrow \bar{E}_{n}$ from the closure of $E_{n}$ into itself and $\tilde{T}$ is the identity map on the faces of $\bar{E}_{n}$.

These assertions imply that $\tilde{T}$, and hence $T$, is surjective; for if $\tilde{T}$ onitted a point of $E_{n}$ one could construct a continuous map $\bar{E}_{n} \rightarrow \partial \bar{E}_{n}$ which is the identity on $\partial \bar{E}_{n}$. But it is a standard theorem of algebraic topology that such a retraction cannot exist [6, Corollary 1.17, p. 27].

We now describe the construction of $\gamma=\gamma(\vec{\varphi})$. We make use of a positive number $r=r(\vec{\varphi})$ which depends continuously on $\vec{\varphi}$ and which is small compared to the distances between the points $1, e^{i \varphi_{1}}, e^{i \varphi_{2}}, \ldots, e^{i \varphi_{n}}$. For example, we may choose

$$
r=\frac{1}{100} \min \left\{\varphi_{1}-0, \varphi_{2}-\varphi_{1}, \varphi_{3}-\varphi_{2}, \ldots, \varphi_{n}-\varphi_{n-1}, 2 \pi-\varphi_{n}\right\} .
$$




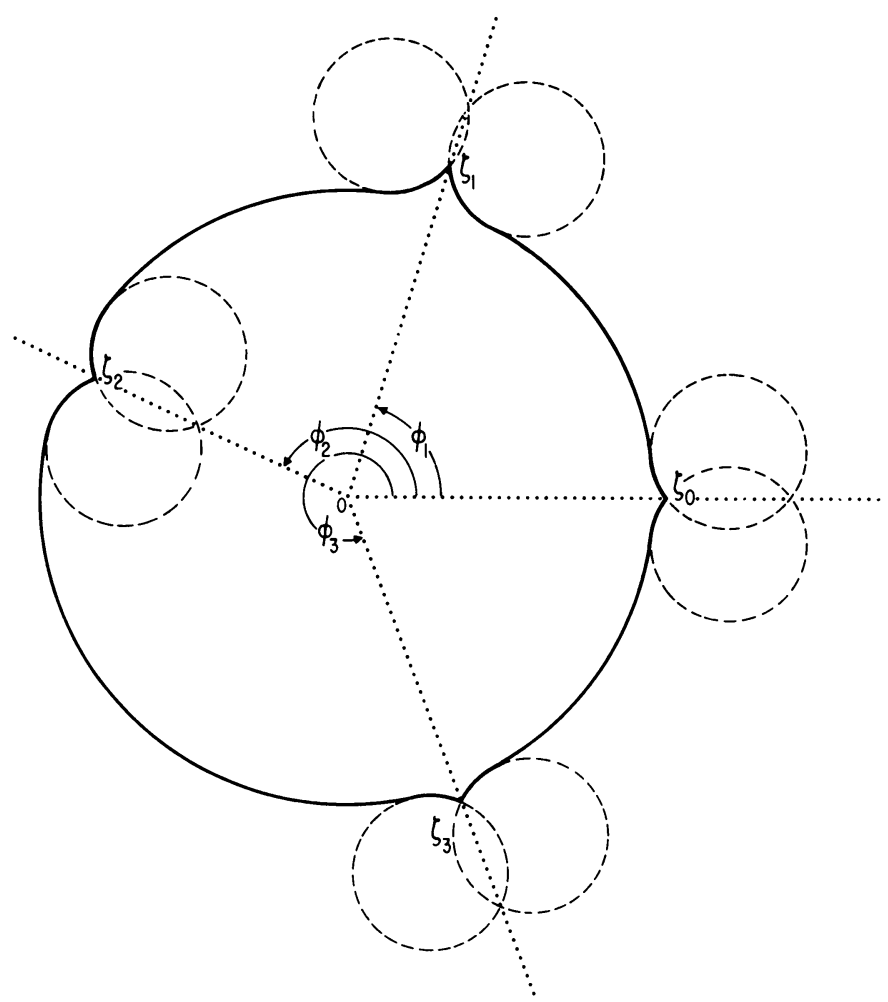

FIGURE 1

The vertices $\zeta_{0}, \zeta_{1}, \zeta_{2}, \ldots, \zeta_{n}$ of $\gamma$ will lie on the rays through the origin which have arguments $0, \varphi_{1}, \varphi_{2}, \ldots, \varphi_{n}$ respectively. Each edge of $\gamma$ will consist of an arc on the unit circle, plus two arcs of circles of radius $r$ which are tangent to the unit circle. Figure 1 should make the construction clear if one recalls that the vertex angles are to be the fixed numbers $\alpha_{0}, \alpha_{1}, \ldots, \alpha_{n}$ which do not depend on $\vec{\varphi}$.

To prove the first assertion let $\vec{\varphi}_{j} \rightarrow \vec{\varphi}$ in $E_{n}$. Then $\gamma\left(\vec{\varphi}_{j}\right)$ converges to $\gamma(\vec{\varphi})$ in the sense of Fréchet; that is, there are parametrizations

$$
t \mapsto h_{j}(t), \quad t \mapsto h(t) \quad(t \in[0,1])
$$

of $\gamma\left(\vec{\varphi}_{j}\right)$ and $\gamma(\vec{\varphi})$, respectively, with the property that $h_{j}$ converges to $h$ uniformly on $[0,1]$. Let $F_{j}$ and $F$ denote the Riemann mapping functions for the insides of $\gamma\left(\vec{\varphi}_{j}\right)$ and $\gamma(\vec{\varphi})$ normalized by $0 \mapsto 0$ and $\zeta_{0} \rightarrow 1$. Let $G_{j}=F_{j}^{-1}$ and $G=F^{-1}$. Then $G_{j}$ and $G$ can be extended to homeomorphisms $\tilde{G}_{j}$ and $\tilde{G}$ of $\{|z| \leq 1\}$ onto the closures of Jordan regions. Since the boundaries $\gamma\left(\vec{\varphi}_{j}\right)$ and $\gamma(\vec{\varphi})$ of these regions converge in the sense of Fréchet, one can apply Rádo's theorem [4] (see also Tsuji $[\mathbf{5}$, p. $383 \mathrm{ff}$.$] , Courant [\mathbf{2 , 3}])$ to conclude that $\tilde{G}_{j}$ converges uniformly to $\tilde{G}$ on $\{|z| \leq 1\}$. It follows that if $\zeta_{0}^{j}, \zeta_{1}^{j}, \ldots, \zeta_{n}^{j}$ denote the vertices of $\gamma\left(\vec{\varphi}_{j}\right)$ and $\varsigma_{0}, \zeta_{1}, \ldots, \zeta_{n}$ denote the vertices of $\gamma(\vec{\varphi})$, then $F_{j}\left(\zeta_{k}^{j}\right) \rightarrow F\left(\varsigma_{k}\right)$ for $k=1,2, \ldots, n$. This means $T\left(\vec{\varphi}_{j}\right) \rightarrow T(\vec{\varphi})$ in $E_{n}$, and hence, $T: E_{n} \rightarrow E_{n}$ is continuous.

The proof of the second assertion is based on the same convergence principle used above. Let $\left\{\vec{\varphi}_{j}\right\}$ be a sequence in $E_{n}$ which tends to a boundary point $\vec{\varphi}$ 
of $E_{n}$. Then $r\left(\vec{\varphi}_{j}\right) \rightarrow 0$; the construction of $\gamma$ shows that $\gamma\left(\vec{\varphi}_{j}\right)$ approaches the unit circle $\partial \Delta$ (in the sense of Fréchet) and the vertices $\zeta_{k}^{j}$ approach the points $e^{i \varphi_{k}}(k=0,1, \ldots, n)$ where $\vec{\varphi}=\left(\varphi_{1}, \varphi_{2}, \ldots, \varphi_{n}\right)$ and $\varphi_{0} \equiv 0$. The Radó theorem implies that the normalized Riemann mapping function $F_{j}$ on the inside of $\gamma\left(\vec{\varphi}_{j}\right)$ has an inverse which, when extended continuously to $\{|z| \leq 1\}$, converges uniformly to the identity function. Hence $T\left(\vec{\varphi}_{j}\right)$ approaches $\left(\varphi_{1}, \varphi_{2}, \ldots, \varphi_{n}\right)=\vec{\varphi}$. Thus the continuous extension $\tilde{T}$ of $T$ is the identity on the faces of $\bar{E}_{n}$.

\section{REFERENCES}

1. R. Courant, Über eine Eigenschaft der Abbildungsfunktionen bei konformer Abbildung, Nachrichten von der Gesellschaft der Wissenschaften, Göttingen, 1914, pp. 101-109.

2. __ Bemerkung zu meiner Arbeit. "Über eine Eigenschaft. ..", Nachrichten von der Gesellschaft der Wissenschaften, Göttingen, 1922, pp. 69-70.

3. G. M. Goluzin, Geometric theory of functions of a complex variable, Transl. Math. Monos., Vol. 26, Amer. Math. Soc., Providence, R. I., 1969, 676 pp.

4. T. Radó, Sur la représentation conforme des domaines variables, Acta Litt. Sci. Univ. FranciscoJosephinae 1 (1923), 180-186.

5. M. Tsuji, Potential theory in modern function theory, Maruzen, Tokyo, 1959, $590 \mathrm{pp}$.

6. J. W. Vick, Homology theory, Academic Press, New York and London, 1973, 237 pp.

Department of Mathematics, University of California, San Diego, La Jolla, CALIFORNIA 92093 